\title{
Differential photometry of speckle-interferometric binary and multiple stars
}

\author{
E. A. Pluzhnik ${ }^{1,2,3}$ \\ ${ }^{1}$ Subaru Telescope, National Astronomical Observatory of Japan, 650 North A'ohoku Place, Hilo, HI 96720, USA \\ e-mail: pea@subaru.naoj.org \\ 2 Special Astrophysical Observatory, Nizhnij Arkhyz, Zelenchuk region, Karachai-Cherkesia, 369167, Russia \\ 3 Institute of Astronomy of Kharkov National University, Sumskaya 35, 61022 Kharkov, Ukraine
}

Received 24 April 2004 / Accepted 3 October 2004

\begin{abstract}
A method for differential photometry of speckle-interferometric binary and multiple stars is presented. Both the accuracy and sources of systematic errors of the method are analysed. The photometric accuracy ranges between 0.02 and $0 \mathrm{~m} 20$, depending on the atmospheric seeing, the brightness and the separation of the system components. A comparison between our magnitude differences and those of other authors is presented.
\end{abstract}

Key words. stars: binaries: visual - techniques: interferometric - techniques: photometric - stars: general

\section{Introduction}

The study of binary stars is the most useful direct way to connect stellar theoretical models with actual observational parameters. The most striking example is the mass-luminosity relation which is completely based on the masses of binary components. The following problems can be addressed with intensive positional and photometric observations of binaries: improving of the mass-luminosity relation, especially for low luminosity stars (Andersen 1991; Henry et al. 1999); spectral classification and colour/effective temperature estimation for components of close binaries (ten Brummelaar et al. 2000); determination of the evolutionary status of components, and the distribution of mass ratios in binary and multiple systems (Preibish et al. 2000; Woitas et al. 2001). Speckle interferometry is currently the main method for the accurate astrometry of binary and multiple stars (Hartkopf et al. 2001). Unfortunately, high-accuracy photometry of individual components with this method still remains difficult (Worley et al. 2001). Among more than 70000 measurements of magnitude differences for binaries included in the Second Photometric Magnitude Difference Catalog (Mason \& Wycoff), only 676 have been made with interferometric techniques. The accuracy of these estimates ranges between 0.1 and 0.5 and is worse than 0.2 for most of them. For stars as faint as $10^{\mathrm{m}}-13^{\mathrm{m}}$ with separations smaller than $0{ }^{\prime} 2-00^{\prime} 3$ it is also difficult to solve the problem with other techniques, especially in visible bands. Interferometry with the Fine Guidance Sensor on the Hubble Telescope (Henry et al. 1999) is currently the only way to make accurate photometry of such binaries.
High-accuracy differential photometry was performed for the first time with the "fork" algorithm by Bagnuolo and Sowell (1988). The method has been succesfully applied to Capella and some bright Hyades binaries (Dombrowski 1989). But similar to other "shift-and-add" based methods, measurements with the "fork" algorithm are possible for bright objects only. This is also true for the "directed vector-autocorrelation" algorithm (Bagnuolo et al. 1992). The uncertainties of routine measurements with this method, as mentioned by Hartkopf et al. (1996), are generally about 0.5 . Other possible ways to solve the problem include:

- photometry with adaptive optics (AO) (ten Brummelaar et al. 1996, 2000, hereafter tB1996 and tB2000) which has an inherent accuracy of $0 . \mathrm{m}_{05} 00^{\mathrm{m}} \cdot 1$ for stars as faint as $3^{\mathrm{m}}-7^{\mathrm{m}}$ (Fig. 3);

- fitting of the visibility function by a model function (Horch et al. 2001, 2004, hereafter H2001 and H2004);

- image restoration (Schöller et al. 1998; Prieur et al. 2003);

- long baseline optical interferometry.

Because of difficulties in solving the inverse problems, the typical uncertainties of the fitting and restoring methods are $0.1-0.2$ for systems that are brighter than $9 \mathrm{~m} 0$ (Figs. 3, 5).

The most numerous photometric measurements of components of multiple stars were made by HIPPARCOS (ESA 1997). However errors of the HIPPARCOS magnitude differences for close pairs which are fainter than $10^{\mathrm{m}}$ are often comparable with measured values themselves. Two-colour relative photometry based on the TYCHO data has been obtained for 5173 relatively bright systems with 

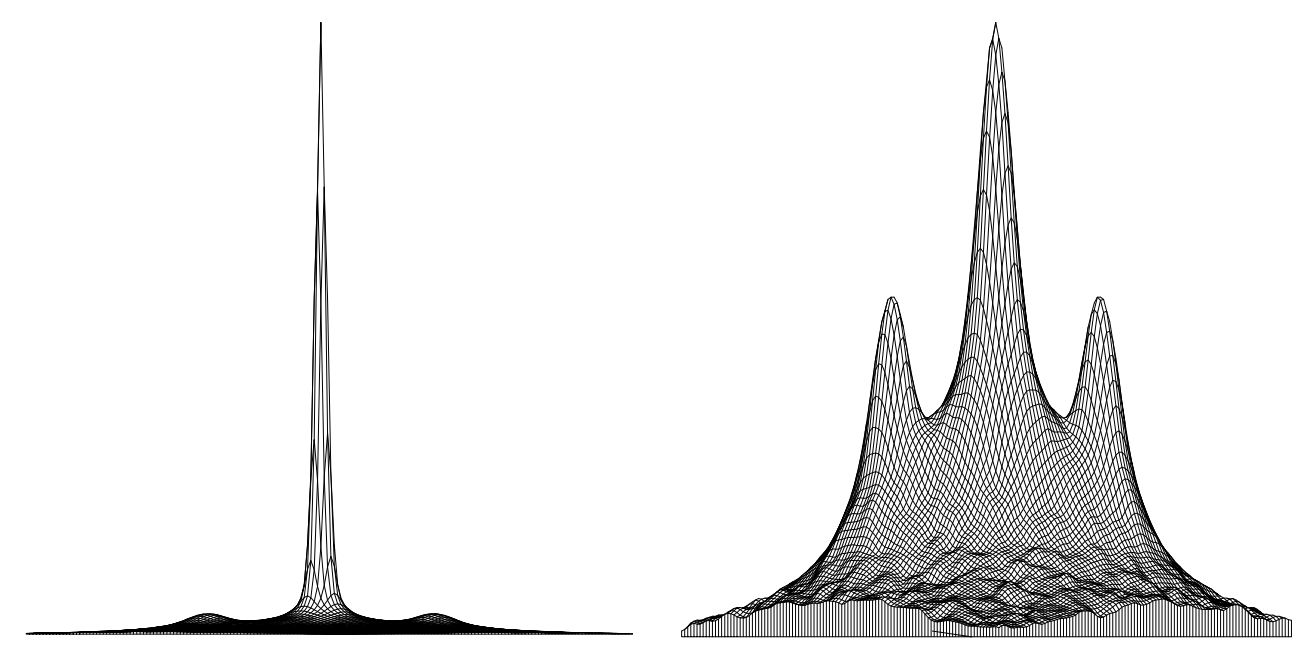

Fig. 1. The autocorrelation of the binary star DF Tau $\left(\rho=0^{\prime}{ }^{\prime} 1, V=12.1, \Delta m=0^{\mathrm{m}} \cdot 23 \pm 0.111, \mathrm{~B} 2004\right)$ without (left) and with (right) the photon bias correction.

separations larger than 0.3 with the "cleaning" algorithm (Fabricius \& Makarov 2000).

In this paper a new method to determine magnitude differences is proposed which is based on a standard power spectrum analysis of speckle series. This method was successfully applied to our speckle-interferometric data obtained in 1998 (Balega et al. 2002, hereafter B2002) and 1999 (Balega et al. 2004, hereafter B2004). Our methodology is explained in Sect. 2. In Sect. 3, we analyse the photometric accuracy, and in Sect. 4 we compare our differential magnitudes with those taken by HIPPARCOS and other authors. The sources of systematic errors and an algorithm to correct magnitude differences for the effect of the limited field of view are also described in Sect. 4.

\section{Measurement of magnitude differences}

Interferometric determination of magnitude differences $\Delta m$ leads to measurements of either the peak amplitude ratio in the object autocorrelation function or the fringe contrast in the object power spectrum/visibility function. With additive noise taken into account, the power spectrum of speckle interferometric frames $\left\langle|I(v)|^{2}\right\rangle$ can be expressed as

$\left\langle|I(v)|^{2}\right\rangle=|O(v)|^{2}\left\langle|S(v)|^{2}\right\rangle+N(v)$,

where $v$ is a spatial frequency vector, $O(v)$ is the Fourier transform of the object intensity distribution, $\left\langle|S(v)|^{2}\right\rangle$ is the speckle interferometric transfer function (STF) and $N(v)$ represents the power spectrum of noisy events. The photon noise (Goodman \& Belsher 1976) and the readout noise predominantly contribute to the function $N(v)=N_{\mathrm{p}}(v)+N_{\mathrm{r}}(v)$. For modern photon-noise-limited detectors the photon bias term $N_{\mathrm{p}}(\boldsymbol{v})$ is much larger than the readout noise term $N_{\mathrm{r}}(v)$, which can be easily corrected. Therefore we consider the photon bias influence only.

Let $N_{\mathrm{p}}(\boldsymbol{v})=N_{0} n_{\mathrm{p}}(\boldsymbol{v})$, where $n_{\mathrm{p}}(\boldsymbol{v})$ is the normalized photon bias term and $N_{0}$ is the photon bias amplitude. The normalized photon bias $n_{\mathrm{p}}(\boldsymbol{v})$ depends on the shape of photon events. It can be determined as the normalized power spectrum of flat field frames. The photon bias amplitude can be obtained from the power spectrum beyond the telescope cut-off frequency, where $|O(v)|^{2}\left\langle|S(v)|^{2}\right\rangle$ is equal to zero. If the photon bias has been corrected, we may deconvolve Eq. (1) and obtain the parameters of the system by a fitting procedure. This is suitable for positional measurements, but it is inappropriate in determining magnitude differences. The main difficulties of deriving $\Delta m$ are the following:

1. the required accuracy of the approximation for the photon bias amplitude is a fraction of a percent (Fig. 1), whereas the usual accuracy is about several percent;

2. we must be sure that the estimate of $n_{\mathrm{p}}(\boldsymbol{v})$, derived from flat field frames, correctly describes the photon bias term in Eq. (1), which might be affected, e.g., by the weak registration nonlinearity;

3. deconvolution is known to be an ill-posed problem.

\subsection{Binary stars. Circularly symmetric STF}

The photon bias changes the contrast of the power spectrum fringes and affects the $\Delta m$ estimate. Let us assume that the STF is circularly symmetric. In this case, we may select an annular area near the spatial frequency $v$ to be narrow enough that $\left\langle|S(v)|^{2}\right\rangle$ may be considered to be constant. If the value of the amplitude $N_{0}$ is fixed, both astrometric and photometric solutions can be obtained in the annular area by least squares fitting with a model function

$F_{2}(v)=\alpha_{v}+\beta_{v} \cos (2 \pi v \rho)$.

In Eq. (2), $\alpha_{v}$ and $\beta_{v}$ represent unknown constants, and $\rho$ is the unknown vector of the system separation. Weighted mean values of the separation $\rho$ and the position angle $\theta$, derived from different annular areas, can be used in the further $\Delta m$ determination.

Let us introduce a contrast function determined as

$C(v)=\frac{2 \alpha_{v}}{\beta_{v}}$. 

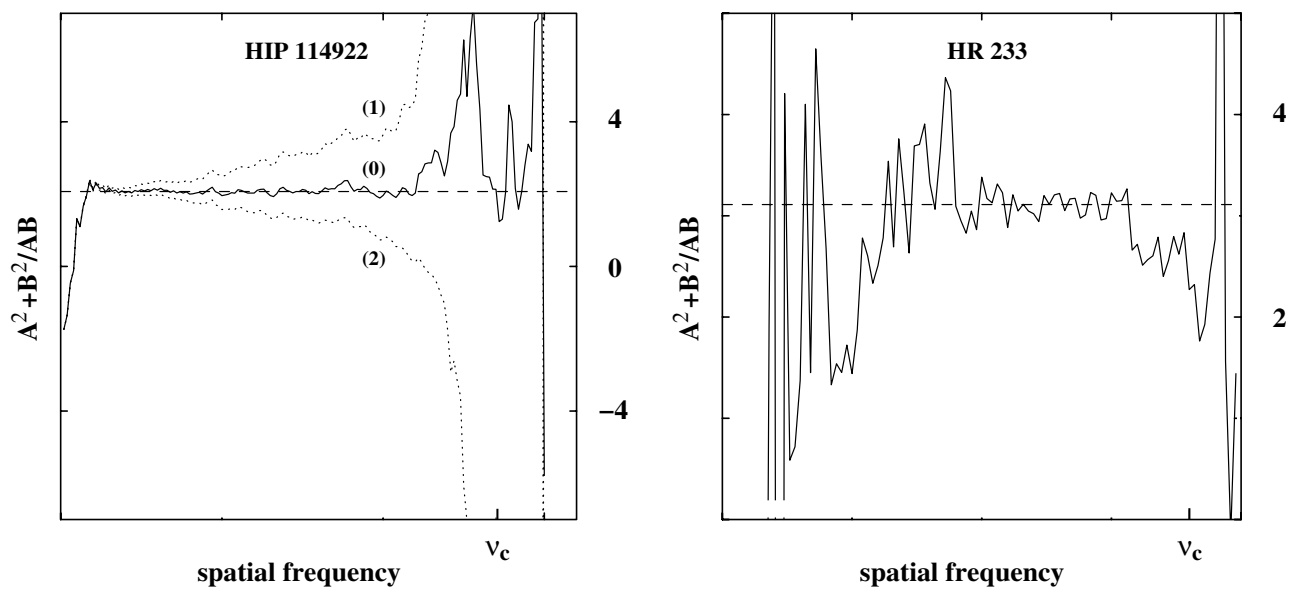

Fig. 2. The contrast function as a function of spatial frequency for binaries HIP $114922\left(\rho=0\right.$ ' $107, V=11^{\mathrm{m}} \cdot 3, \Delta m=0$. $16 \pm 0$. 09 , the photon bias amplitudes $N_{0}^{(1)}=0.93 N_{0}^{(0)}$ and $N_{0}^{(2)}=1.04 N_{0}^{(0)}$, B2004) and HR $233\left(\rho=0 \prime^{\prime} 0160 \pm 0.0005, V=5 \cdot 4, \Delta m=1 \mathrm{~m} \cdot 08 \pm 0.08\right.$, B2004). The telescope cut-off limit $v_{\mathrm{c}}$ is shown.

For a binary with component brightnesses $\mathrm{A}$ and $\mathrm{B}$, the contrast function $C(v)$ can be written as

$C(v)=\frac{A^{2}+B^{2}}{A B}=\frac{A}{B}+\frac{B}{A}$

and must remain constant for all annular areas. However, when the adopted value of the amplitude $N_{0}$ differs from its true value, the estimate of the contrast function turn out to be frequency-dependent. Thus, if the amplitude $N_{0}$ is overestimated, the contrast function decreases as the frequency grows, otherwise, the contrast function increases with frequency increasing (Fig. 2). It is clear that the correct value of the amplitude $N_{0}$ can be found from the condition $\mathrm{d} C(v) / \mathrm{d} v=0$. This must be true within some spatial frequency range, excluding the lowest frequencies where the effect of atmospheric seeing is strong, and the highest ones where the signal-to-noise ratio is low.

The value of $\mathrm{d} C(v) / \mathrm{d} v$ can be determined as a slope of the weighted first order least squares fitting for $C(v)$ :

$C(v)=C_{0}+C_{1} v$

where $C_{0}$ and $C_{1}$ are unknown parameters, and weights of the equations are selected according to the relative error of the coefficient $\beta_{v}$.

If we choose the amplitude $N_{0}$ according to the condition $\mathrm{d} C(v) / \mathrm{d} v=C_{1}=0$, then the intensity ratio of the components $A / B$ can be obtained using the equation:

$\frac{A^{2}+B^{2}}{A B}=\frac{A}{B}+\frac{B}{A}=C_{0}$,

and thus

$\Delta m=m_{A}-m_{B}=-2.5 \log (A / B)$.

The error of the magnitude difference $\sigma_{\Delta m}$ can be obtained from the error of the parameter $C_{0}$ in the usual way (Kuzmenkov 1986).

\subsection{Binary stars. Noncircular STF}

The proposed method can be easily generalized to the case of a noncircular STF. Note that we have nowhere used the circular symmetry of the STF, but only supposed the STF to be constant within the spatial frequency areas selected for the approximation. Therefore the proposed method is applicable for any STF provided that circular annular areas are replaced with areas where the STF may be considered as a constant.

In most cases one may restrict the model to an "elliptical" STF, i.e. one which may be considered as a constant within elliptical annular areas. Geometric parameters of the areas where the STF is constant (CTFA - constant transfer function area), as well as the vector of the system separation, can be determined simultaneously to the iteration procedure in which the successive estimate of $\rho$ is obtained in a manner similar to the case of a circular STF with substitution of circular CTFAs for elliptical ones. The geometry of a CTFA can be determined from the condition that the correlation index $k$ between the power spectrum and the function $\cos \left(2 \pi v \rho_{0}\right)$ in the CTFA be maximal:

$k=\frac{\overline{\left(\left\langle|I(\boldsymbol{v})|^{2}\right\rangle-I^{(2)}\right)\left(\cos \left(2 \pi v \rho_{0}\right)-\langle\cos \rangle\right)}}{\sigma_{\mathrm{p}} \sigma_{\cos }}$.

In Eq. (8) the overline indicates an average, $\rho_{0}$ is the previous estimate of the separation vector, $I^{(2)},\langle\cos \rangle, \sigma_{\mathrm{p}}$ and $\sigma_{\cos }$ are average values and rms deviations of the power spectrum and the function $\cos \left(2 \pi v \rho_{0}\right)$ in the CTFA, respectively. Such an approach gives reliable results while estimates of $\rho$ and $\Delta m$ remain uncorrelated $(\rho>2 \lambda / D, D$ is the telescope diameter and $\lambda$ is the wavelength, Kuzmenkov 1986). At smaller separations a correlation between the calculated CTFA parameters and $\rho$ appears, and the method becomes inapplicable. To determine the CTFAs in this case, a reference star should be used, selected either from single stars or sufficiently wide binaries.

In Fig. 2 the contrast function for the system HR 233 (B2004) is presented as an example of measurement for the extremely close binary $\left(\rho=0{ }^{\prime} 0160 \pm 0.0005, \theta=273^{\circ} .0 \pm 1.5\right.$, $\Delta m=1.08 \pm 0.08$, the telescope diffraction limit is $0 .^{\prime} 02$ ). The confidence of the measurement is confirmed by comparing 
the positional parameters of the system with the known orbit (Mason et al. 1997). The residuals of our measurement from the orbit are 0.0027 and 11.6 in $\rho$ and $\theta$, respectively. The measured point has the smallest separation in comparison with other mesurements of the star. Nevertheless the point lies on the orbit better than most of the other ones. The binary HR 640 ( $\left.\rho=0 .{ }^{\prime} 0946 \pm 0.0003\right)$ was used as the reference source.

\subsection{Triple stars}

For a triple star, we can obtain the system parameters following the same idea of estimating them within CTFAs. However, there are some significant differences, namely:

1. the six secondary peaks of the autocorrelation function contain complete information about positional and photometric parameters of the triple. This makes it possible to obtain the photon noise amplitude in any CTFA using a standard fitting procedure;

2. there are two additional relations between the system parameters:

$$
\left\{\begin{array}{l}
\Delta m_{12}+\Delta m_{23}+\Delta m_{31}=0 \\
\boldsymbol{r}_{12}+\boldsymbol{r}_{23}+\boldsymbol{r}_{31}=0,
\end{array}\right.
$$

where $\Delta m_{i j}$ and $\boldsymbol{r}_{i j}$ are, respectively, the magnitude difference and the vector of separation between the $i$ th and $j$ th components.

With all the above taken into account, the model function can be written in the form

$$
\begin{aligned}
F_{3}(v)= & \frac{V_{12} V_{31}}{V_{23}}+\frac{V_{12} V_{23}}{V_{31}}+\frac{V_{23} V_{31}}{V_{12}} \\
& +V_{12} \cos \left(2 \pi v \rho_{12}\right)+V_{23} \cos \left(2 \pi v \rho_{\mathbf{2 3}}\right) \\
& +V_{31} \cos \left(2 \pi v \rho_{\mathbf{3 1}}\right)+C_{\mathrm{p}} n_{\mathrm{p}}(\boldsymbol{v}),
\end{aligned}
$$

where $V_{i j}=A_{i} A_{j}$, and $A_{i}$ is the intensity of the ith component. A possible way to solve the fitting problem is to use the iteration process consisting of the following steps:

1. selection of CTFAs in a manner similar to the case of binaries;

2. determination of amplitudes $V=V_{12} V_{31} / V_{23}+$ $V_{12} V_{23} / V_{31}+V_{23} V_{31} / V_{12}, \quad V_{i j}, \quad C_{\mathrm{p}}$ and $\Delta m_{i j}^{\prime}=$ $-2.5 \log V_{k i} / V_{j k}$ within each CTFA by approximation of the power spectrum with a linear model, using previously estimated positions;

3. estimation of positional parameters $\rho_{i j}{ }^{\prime}=\left(x_{i j}^{\prime}, y_{i j}^{\prime}\right)$ for each CTFA, using measured amplitudes $V, V_{i j}$ and $C_{\mathrm{p}}$;

4. obtaining successive $\Delta m_{i j}$ and $\rho_{i j}$ values by the weighted least squares solution of the following equation systems, written for all selected CTFAs:

$$
\begin{aligned}
& \left\{\begin{array}{l}
\boldsymbol{r}_{12}=\boldsymbol{r}_{12}^{\prime} \\
\boldsymbol{r}_{23}=\boldsymbol{r}_{23}^{\prime} \\
\boldsymbol{r}_{12}+\boldsymbol{r}_{23}=-\boldsymbol{r}_{31}^{\prime},
\end{array}\right. \\
& \left\{\begin{array}{l}
\Delta m_{12}=\Delta m_{12}^{\prime} \\
\Delta m_{23}=\Delta m_{23}^{\prime} \\
\Delta m_{12}+\Delta m_{23}=-\Delta m_{31}^{\prime} .
\end{array}\right.
\end{aligned}
$$

Weights of the equations for each of two systems are selected accoding to the rms errors of the $\rho_{i j}{ }^{\prime}$ or $\Delta m^{\prime}$ estimates. Errors $\sigma_{\Delta m_{i j}}$ can be obtained using the standard least squares approach.

\subsection{Errors of positional parameters}

Since a correlation between parameters of the nearby CTFAs exists, as well as a correlation between the CTFA parameters and $\boldsymbol{\rho}^{\prime}$, formal errors $\sigma_{\rho}$ and $\sigma_{\theta}$ appear to be much less than their actual values.

Actual errors of positional parameters can be estimated as rms deviations of these parameters taken within each CTFA. In the case of binaries this results in:

$\sigma_{\rho}=\frac{\sum\left(\rho-\rho_{i}\right)^{2} / \sigma_{\rho_{i}}^{2}}{\sum 1 / \sigma_{\rho_{i}}^{2}} \quad \sigma_{\theta}=\frac{\sum\left(\theta-\theta_{i}\right)^{2} / \sigma_{\theta_{i}}^{2}}{\sum 1 / \sigma_{\theta_{i}}^{2}}$,

For triple stars errors can be written in the form

$\sigma_{\rho_{i j}}^{2}=\frac{\sigma_{x_{i j}}^{2} x_{i j}^{2}+\sigma_{y_{i j}}^{2} y_{i j}^{2}}{\rho_{i j}^{2}} \quad \sigma_{\theta_{i j}}^{2}=\frac{\sigma_{x_{i j}}^{2}+\sigma_{y_{i j}}^{2}-\sigma_{\rho_{i j}}^{2}}{\rho_{i j}^{2}}$,

where

$\sigma_{x_{i j}}^{2}=\frac{\sum\left(x_{i j}-x_{i j}^{\prime}\right)^{2} / \sigma_{x_{i j}}^{2}}{\sum 1 / \sigma_{x_{i j}}^{2}}$

and

$\sigma_{y_{i j}}^{2}=\frac{\sum\left(y_{i j}-y_{i j}^{\prime}\right)^{2} / \sigma_{y_{i j}}^{2}}{\sum 1 / \sigma_{y_{i j}}^{2}}$.

Summation is carried out for all selected CTFAs.

These accuracy evaluations take into account uncertainties of the CTFAs' determination as well as random errors of the power spectrum.

\subsection{Absolute quadrant determination}

When we try to identify from the power spectrum analysis which component of a binary is primary and which is secondary, the problem of the absolute quadrant determination arises (Bagnuolo et al. 1992). High accuracy measurements of $\Delta m$ make it possible to eliminate the $180^{\circ}$ uncertainty in $\theta$ value by using Walker's idea (1978). If we multiply each speckle frame by a function which changes monotonically in the direction from the primary component to the secondary one, $\Delta m$ will be changed too. The true quadrant can be obtained by analysing the sign of $\Delta m$ changes depending on the direction of the function growth. The method gives reliable results for separations exceeding $2 \lambda / D$.

\section{Statistics and accuracy of $\Delta m$ measurements}

We applied the above method to measure multiple star parameters using our speckle interferometric data obtained in 1998 and 1999 (B2002, B2004). The atmospheric seeing varied from 1 .' 2 to $2^{\prime \prime}$ during observational sets. The quantum efficiency of the detector was about $1.5 \%$ and $5.0 \%$ at spectral 

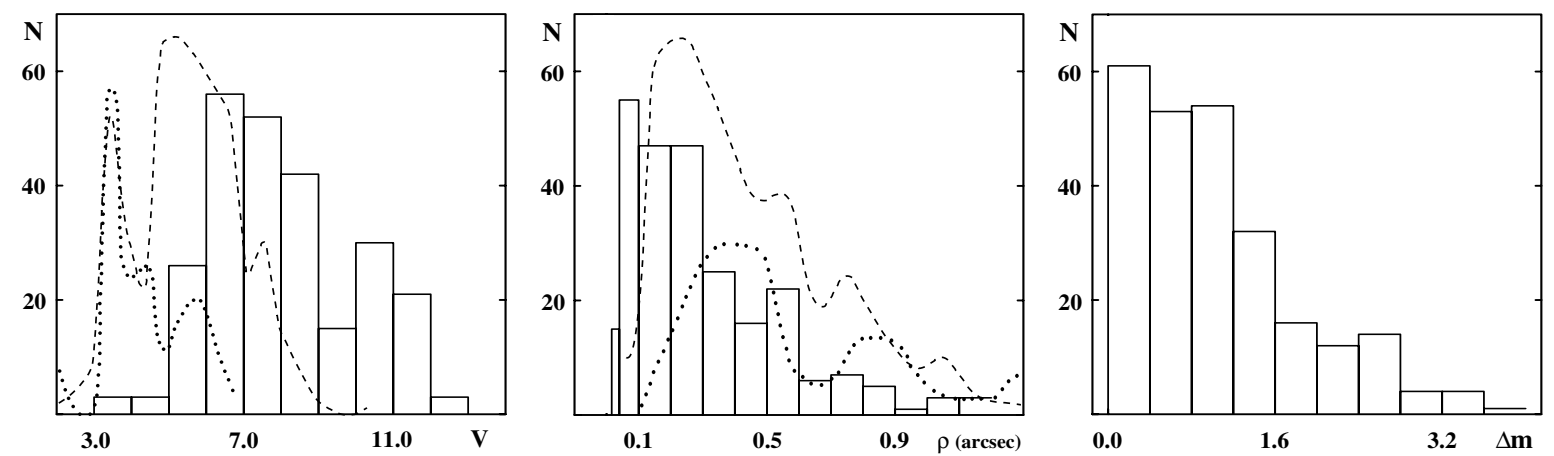

Fig. 3. Statistical distributions for speckle interferometric observations in 1998 and 1999 (B2002, B2004). The number of measurements are ploted vs. visual magnitude $V$, angular separation $\rho$ and magnitude difference $\Delta m$. For comparison, distributions for AO data by tB1996, tB2000 (dotted) and unnormalized distributions for speckle data by H2001, H2004 (dashed) are also presented.
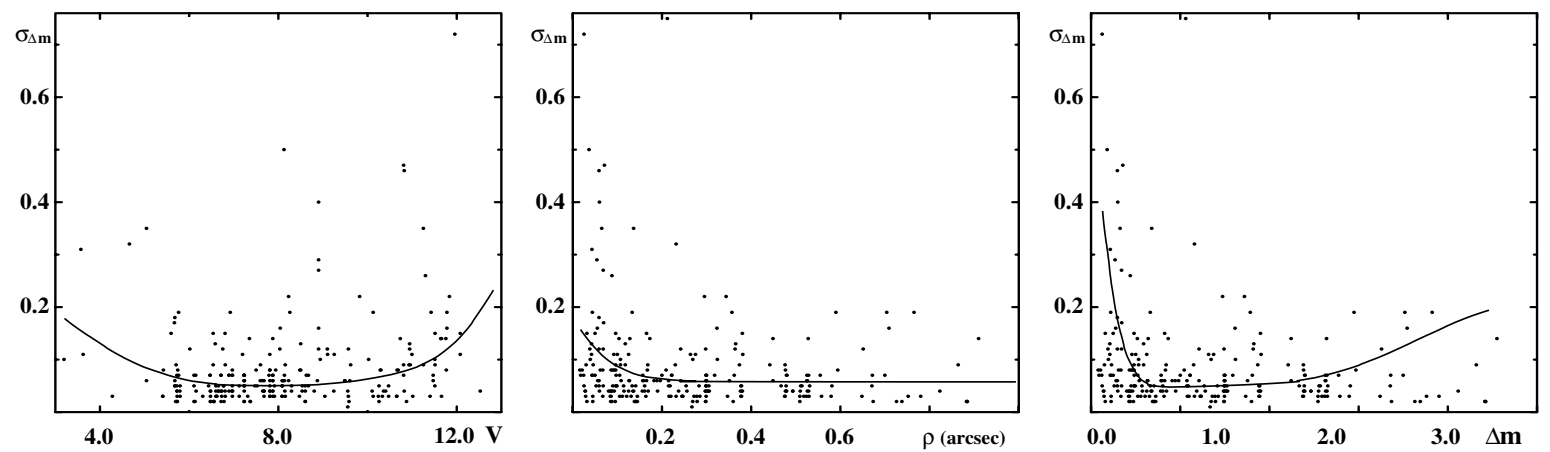

Fig. 4. Errors of $\Delta m$ measurements in 1998 and 1999 (B2002, B2004) vs. visual magnitude $V$, angular separation $\rho$ and magnitude difference $\Delta m$.

bands near $800 \mathrm{~nm}$ and $545 \mathrm{~nm}$ respectively. Separations of the program stars, ranging from $2^{\mathrm{m}}$ to $13^{\mathrm{m}}$ in magnitude, lie within 0 .016 and 1'.6, and their magnitude differences vary from 0 to 3.7 (Fig. 3). As a result, 251 measurements for $\Delta m$ have been made with uncertainties of $0.02-0.20$ (Fig. 5) depending on the atmospheric seeing, the brightness and the separation of the system components. The achieved accuracy of $\Delta m$ measurements plotted vs. the magnitude, separation and magnitude difference of each system is shown in Fig. 4. Note that the accuracy falls towards small $\Delta m$, as well as towards large magnitude differences. The median value of the accuracy is about 0 . 06 .

Initial consistency of the measurements has been tested by comparing the data obtained during different observational sets (Fig. 7). It is easily seen that the value $\eta=$ $\left(\Delta m_{1}-\Delta m_{2}\right) / \sqrt{\sigma_{1}^{2}+\sigma_{2}^{2}}$ must have the Gaussian distribution $\exp \left(-\eta^{2} / 2\right) / \sqrt{2 \pi}$ (Fig. 6), where $\Delta m_{1}$ and $\Delta m_{2}$ are magnitude differences of a star obtained in different observational sets with errors $\sigma_{1}$ and $\sigma_{2}$. We estimated $\bar{\eta}=0.113$ and $\sigma_{\eta}=0.934$ using 34 stars which were observed in 1999 as well as in 1998. These values support statistical hypotheses that $\bar{\eta}=0$ and $\sigma_{\eta}=1$, at significance levels $47 \%$ and $60 \%$ respectively. The average difference of $\Delta m$ values in 1998 and 1999 is equal to 0 m 004 and does not exceed the minimal error of our measurements. Different seeing conditions during observations are assumed to be the reason for this shift. The statistical analysis confirms the high self-consistency of our measurements and the validity of estimated errors.

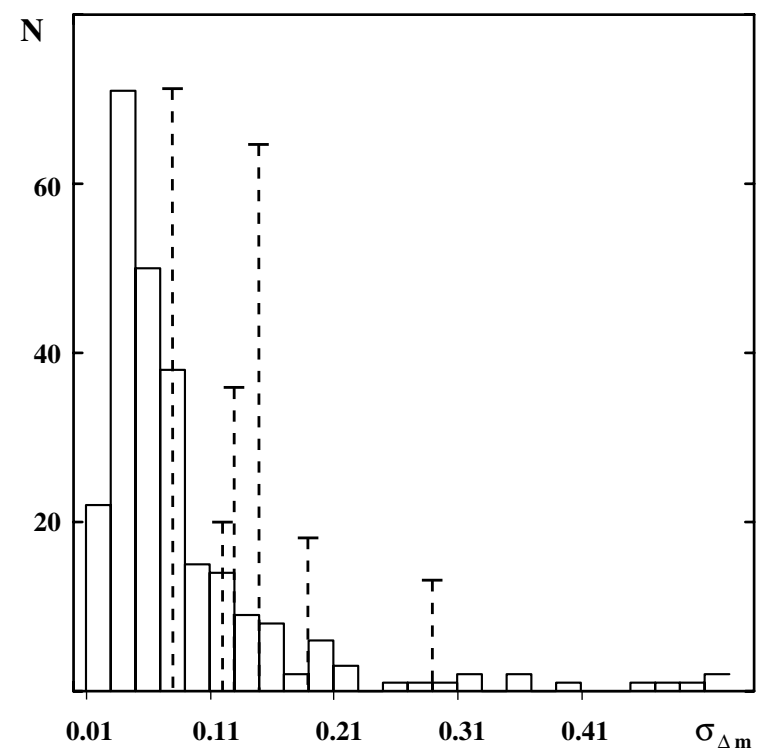

Fig. 5. The distribution of $\Delta m$ errors in 1998 and 1999 (B2002, B2004). The unnormalized distribution of $\Delta m$ errors for $\mathrm{H} 2004$ data is dashed.

\section{Comparison with other measurements}

\subsection{Comparison with HIPPARCOS data}

The reliability of the data was also examined by comparing our results with published magnitude differences. Unfortunately, 




Fig. 6. Comparison of $\Delta m$ measurements in 1998 and 1999. The measured (solid) and the Gaussian (dashed) probability distributions $f(\eta)$ for $\eta=\left(\Delta m_{1}-\Delta m_{2}\right) / \sqrt{\sigma_{1}^{2}+\sigma_{2}^{2}}$ values are presented.

the interference filters used in our observations are different from photometric bands used by other authors. Therefore, direct comparison of $\Delta m$ is difficult. We restrict the comparison to our $545 \mathrm{~nm} / 30 \mathrm{~nm}$ spectral band measurements only, because this band is the closest to the HIPPARCOS one.

Only pairs with separations less than 0.'5 were chosen to compare with HIPPARCOS measurements. Although estimates of the accuracy of our measurements are confirmed at a significance level of $50 \%$, it is easy to detect statistically reliable (at a significance level of 90\%) differences between speckle interferometric and HIPPARCOS measurements. Least squares fitting of relations between our $\Delta m$ and HIPPARCOS $\Delta m_{\mathrm{H}}$ in the $545 \mathrm{~nm} / 30 \mathrm{~nm}, 610 \mathrm{~nm} / 20 \mathrm{~nm}$ and $800 \mathrm{~nm} / 60 \mathrm{~nm}$ bands yields

$\Delta m_{\mathrm{H}}=-0.07[ \pm 0.03]+1.04[ \pm 0.03] \Delta m_{545}$

$\Delta m_{\mathrm{H}}=-0.10[ \pm 0.06]+1.13[ \pm 0.05] \Delta m_{610}$

$\Delta m_{\mathrm{H}}=-0.07[ \pm 0.10]+1.34[ \pm 0.11] \Delta m_{800}$

respectively. These relations show clearly that a bias of about 0. 08 exists between the two kinds of data. The reason for such a bias is considered below.

\subsection{Sources of systematic errors}

In addition to random errors, some systematic errors are inherent in the magnitude difference estimation with speckle interferometry. Nonisoplanicity effects, the limited field of view, errors of the $n_{\mathrm{p}}(\boldsymbol{v})$ determination and the flat field correction may all contribute to the $\Delta m$ value.

Comparing speckle-interferometric $\Delta m$ and HIPPARCOS ones, H2001 have shown that nonisoplanicity effects are negligible if $\rho<2 / \omega\left[{ }^{\prime \prime}\right]$, where $\omega$ is the seeing. This condition sets the upper separation limit of unaffected speckle interferometric
$\Delta m$ measurements as low as $2^{\prime \prime}-3^{\prime \prime}$ under common seeing conditions. Although a dependence of the limit on the telescope diameter may be expected, it can be determined for any telescope.

The limited field of view may systematically increase $\Delta m$ due to cutting of interferometric frames. We consider this effect in detail, because it is the main source of systematic errors for our measurements. We split each frame into the following areas (Fig. 8):

1. main area (MA) is the area in which the speckles each contribute to the secondary autocorrelation peaks;

2. exceptional area (EA) is the area in which the speckles have the secondary ones out of the MA; This area is excluded from the consideration because it is impossible to analyse the contribution of the EA to the autocorrelation function;

3. boundary area (BA) is the rest of the frame;

4. working area (WA) is the frame with the EA excluded.

We define window functions $W_{W}$ and $W_{B}$ as

$W_{W}(\boldsymbol{r})= \begin{cases}1 & \text { inside the WA, } \\ 0 & \text { outside the WA }\end{cases}$

$W_{B}(\boldsymbol{r})= \begin{cases}1 & \text { inside the BA, } \\ 0 & \text { outside the BA. }\end{cases}$

Let $S_{W}(\boldsymbol{v})$ and $S_{B}(\boldsymbol{v})$ be power spectra of speckle series $\left\{I(\boldsymbol{r}) W_{W}(\boldsymbol{r})\right\}$ and $\left\{I(\boldsymbol{r}) W_{B}(\boldsymbol{r})\right\}$, respectively. It is easily seen that the weighted sum $S(\boldsymbol{v})=w_{1} S_{W}(\boldsymbol{v})+w_{2}\left[S_{W}(\boldsymbol{v})-S_{B}(\boldsymbol{v})\right]$ keeps the fringe contrast or the ratio of autocorrelation peaks unbiased when $w_{1}=N_{A} A^{2}+N_{B} B^{2}$ and $w_{2}=N_{A}^{\prime} A^{2}+N_{B}^{\prime} B^{2}$. There $A$ and $B$ are average intensities of primary and secondary speckles, $N_{A}$ and $N_{B}$ are numbers of the primary and secondary speckles in the BA contributing to the secondary autocorrelation peaks, and $N_{A}^{\prime}$ and $N_{B}^{\prime}$ are numbers of the speckles in the BA not contributing to the secondary peaks. The ratio of weights $w_{1}$ and $w_{2}$ may be roughly estimated as

$\frac{w_{1}}{w_{2}}=\frac{\int_{I I}\langle I(\boldsymbol{r})\rangle \mathrm{d} \boldsymbol{r}}{\int_{I}\langle I(\boldsymbol{r})\rangle \mathrm{d} \boldsymbol{r}}$,

where $\langle I(\boldsymbol{r})\rangle$ is the average image, $I$ and $I I$ are two areas near the frame boundary separated by the vector $\rho$ (Fig. 8). The power spectrum $S(v)$ determined in such a way can be used to obtain the unbiased $\Delta m$ value.

In Fig. 7 we present our $\Delta m_{545}^{\mathrm{c}}$ corrected with the algorithm described above, together with magnitude differences by other authors. The least squares fitting of the relation between corrected $\Delta m_{545}^{\mathrm{c}}$ and HIPPARCOS $\Delta m_{\mathrm{H}}$ yields

$\Delta m_{\mathrm{H}}=-0.03[ \pm 0.03]+1.02[ \pm 0.03] \Delta m_{545}^{\mathrm{c}}$.

Taking into account errors of HIPPARCOS and our data, as well as the difference in spectral bands, the consistency between these kinds of data may be regarded as excellent.

Differences between $\Delta m_{545}^{\mathrm{c}}$ and $\Delta m_{\mathrm{H}}$ as functions of the system brightness, separation and $\Delta m$ were examined too. These dependencies are plotted in Fig. 9 and support estimates of the accuracy and the usability range presented above for separations ranging between $0 .{ }^{\prime} 1$ and $0 .{ }^{\prime} 8$. 

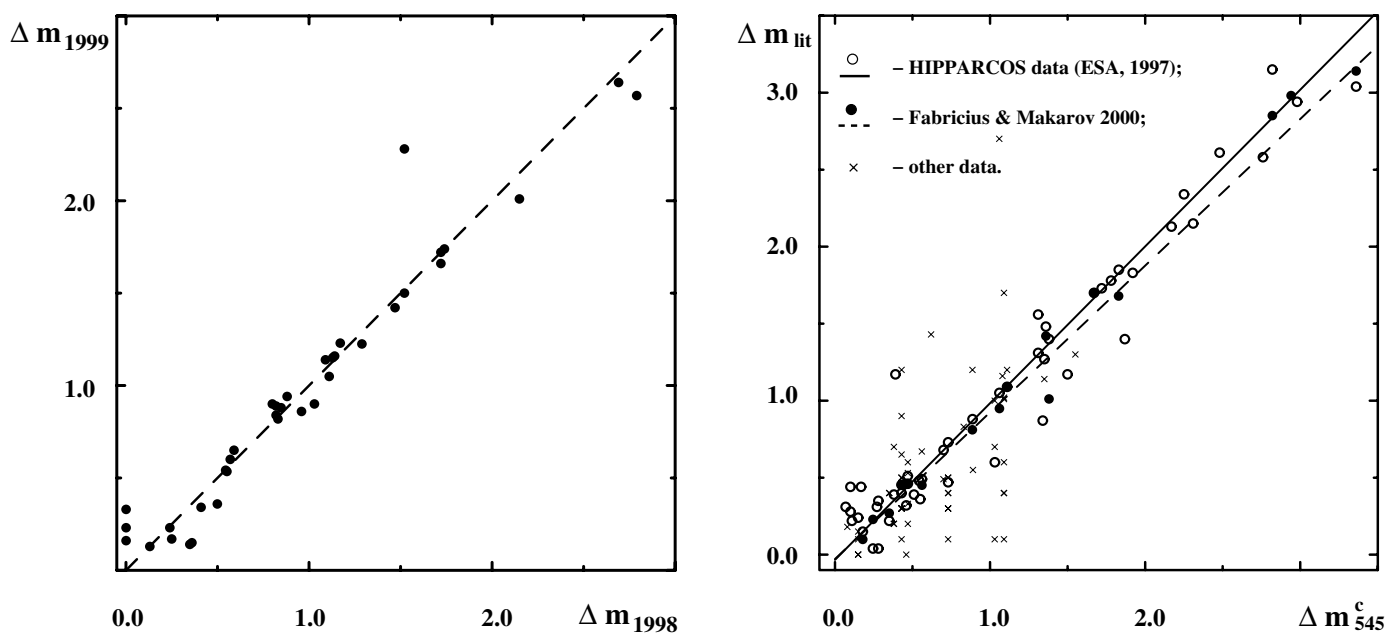

Fig. 7. Comparison of $\Delta m$ measurements in 1998 and 1999 (left $)$ and $\Delta m_{545}^{\mathrm{c}}$ with published data $\Delta m_{\mathrm{lit}}(r i g h t)$. Data of $\mathrm{H} 2001$ and $\mathrm{H} 2004$ are not presented here.

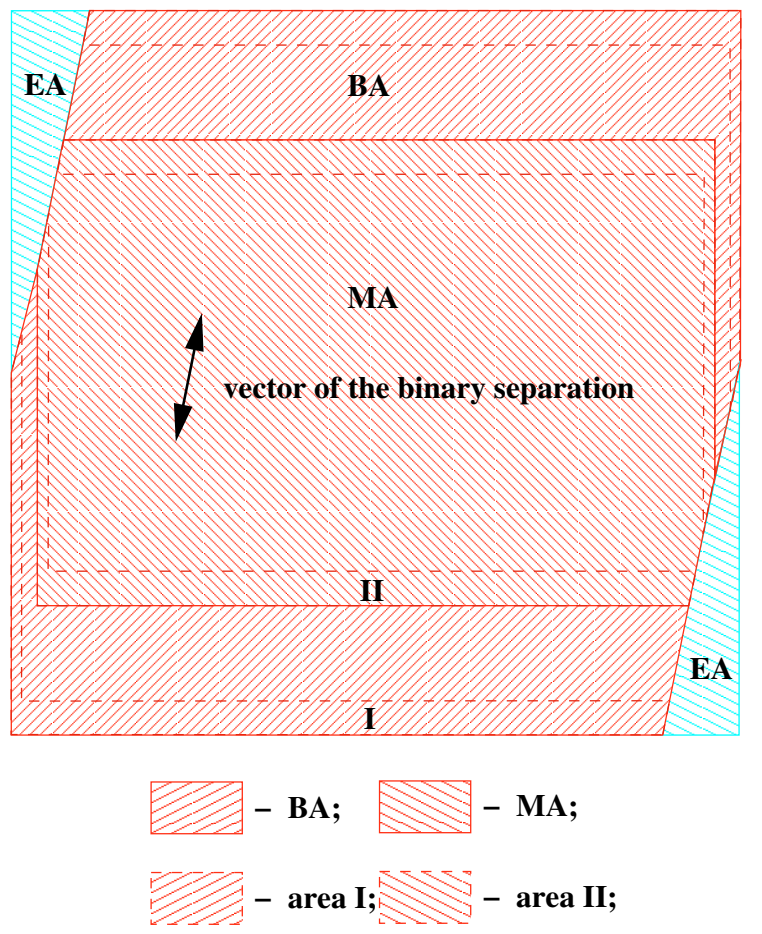

Fig. 8. Area definitions to correct for the effect of the limited field of view.

All the corrected $\Delta m_{545}^{\mathrm{c}}$ values are listed in Table 1 together with the original (B2002, B2004) and HIPPARCOS measurements.

\subsection{Comparison with $A O$ results}

ADS 3475 is the only binary in our list that has been studied with the AO technique as well (tB2000). AO differential magnitudes of the star $(0.15 \pm 0.04$ and $0.20 \pm 0.04$ in $V$ and $I$ bands) are in good agreement with our results $(0.15 \pm 0.10$ and $0.33 \pm 0.09$ in the $545 \mathrm{~nm} / 30 \mathrm{~nm}$ and $800 \mathrm{~nm} / 60 \mathrm{~nm}$ bands, the last value has not been corrected for the field of view effect).
Although it is impossible to compare both methods now, their main advantages are clearly apparent (Fig. 3), namely:

1. published $\mathrm{AO}$ measurements are limited to objects brighter than 6.5 in visible wavelengths, while our approach can be used for stars as faint as $12 \mathrm{~m}$;

2. our method is applicable to separations up to the diffraction limit of the telescope (sometimes even smaller), but it is difficult to measure binaries with separation less than $2 \lambda / D$ with $\mathrm{AO}$. Note that application of the presented approach to AO photometry can improve both the accuracy and the resolution of $\mathrm{AO}$ measurements;

3. the accuracy of $\mathrm{AO}$ measurements for $\Delta m<0$. 2 can be notably higher in comparison with speckle measurements because of the singularity of the solution in Eq. (6) (Kuzmenkov 1986; H2001);

4. AO differential magnitudes can go as deep as $9^{\mathrm{m}}$, while speckle interferometric $\Delta m$ are limited by only $4^{\mathrm{m}}$. At present this advantage of AO has been implemented for separations larger than 0.'4-0.'5 (Turner et al. 2001, 2003).

\subsection{Comparison with other speckle measurements}

Another possibility to test our photometry is speckle interferometric results obtained by Horch et al. (H2001; H2004) with two 60-cm telescopes at Las Campanas and the Cerro Tololo Inter-American Observatory, and the 3.5-m WIYN telescope at Kitt Peak Observatory during the period 1997-2000. A fast readout CCD camera with a quantum efficiency of 30\%-40\% and readout noise of about $10 \mathrm{e}^{-}$was used for these observations. The authors used the standard deconvolution approach to derive $\Delta m$ from speckle data. They obtained more than 800 measurements of $\Delta m$ for stars brighter than 9.5 with random errors of about 0 . 13 (Fig. 3).

We compare only our measurements in the $545 \mathrm{~nm} / 30 \mathrm{~nm}$ band, because our data in other spectral bands have not been corrected for the field of view effects yet. Only 8 stars can be selected that were observed in our $545 \mathrm{~nm} / 30 \mathrm{~nm}$ band as well as in the $503 \mathrm{~nm}$ band used by H2004. Apart from that, we 

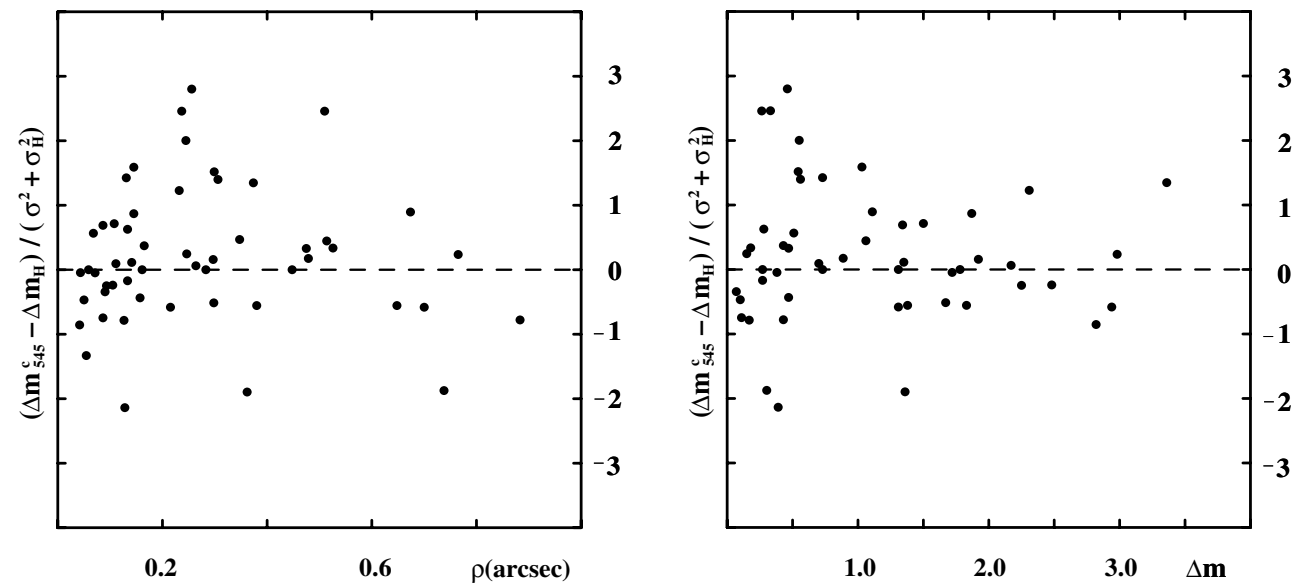

Fig. 9. Comparison of $\Delta m_{545}^{\mathrm{c}}$ with HIPPARCOS measurements. Values $\left(\Delta m_{545}^{\mathrm{c}}-\Delta m_{\mathrm{H}}\right) /\left(\sigma^{2}+\sigma_{\mathrm{H}}^{2}\right)^{1 / 2}$ are ploted vs. angular separation $(l e f t)$ and magnitude difference (right).
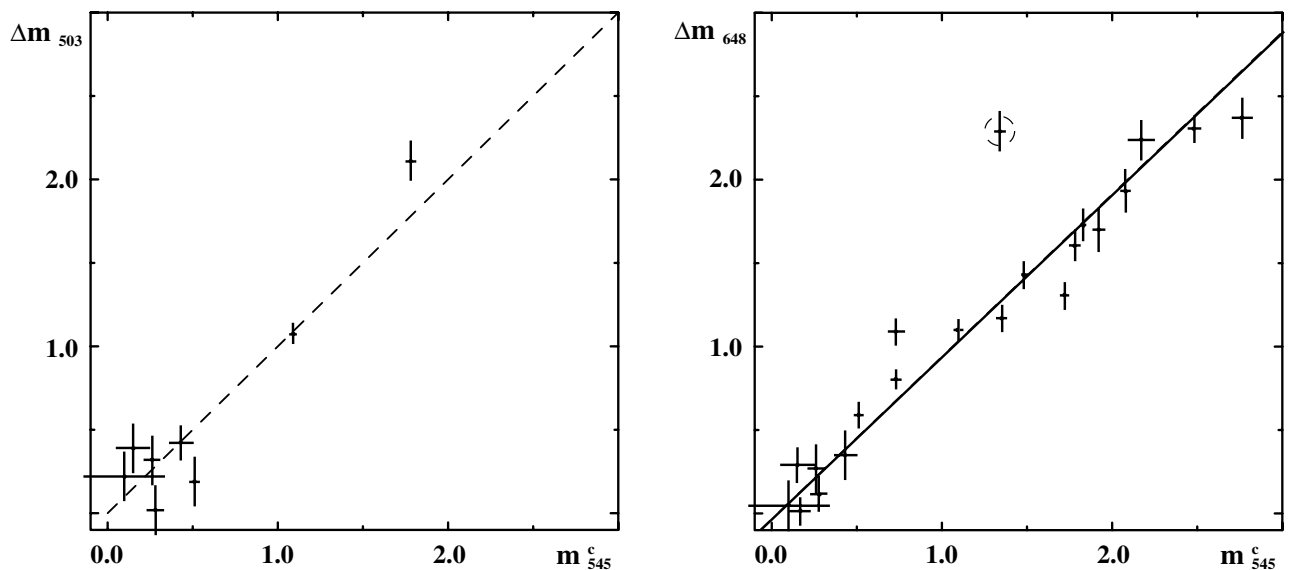

Fig. 10. Comparison of our $\Delta m_{545}^{\mathrm{c}}$ with $\Delta m_{503 / 648}$ measurements by H2001, 2004. Binary HIP 27758 is marked with a dashed circle.

have 22 systems observed by $\mathrm{H} 2004$ in the $648 \mathrm{~nm}$ band also. Though measurements in the $545 \mathrm{~nm}$ and $503 \mathrm{~nm}$ bands seem to be in agreement (Fig. 10), it is difficult to make any statistical conclusion because of the small number of points. The comparison between measurements in the $545 \mathrm{~nm}$ and $648 \mathrm{~nm}$ bands (excluding the HIP 27758 system) yields (Fig. 10)

$\Delta m_{545}=0.04[ \pm 0.07]+1.03[ \pm 0.05] \Delta m_{648}$.

It should be noted that this equation does not show noticable color differences between both sets of measurements and indirectly supports the conclusion by $\mathrm{H} 2004$ about systematic differences between their speckle and AO results. However the statistics are still not strong enough to make a final conclusion.

\section{Summary}

A new method to determine magnitude differences in speckle interferometric binary and multiple stars based on conventional power spectrum estimations has been developed. This method provides high accuracy of the photon bias correction, which is neccesary to obtain precise parameters for speckle interferometric binaries and multiple stars as bright as $11^{\mathrm{m}}-12^{\mathrm{m}}$. The errors of measurements lie between $0^{\mathrm{m}} 02$ and 0.20 , depending on the atmospheric seeing, as well as on the brightness and the separation of the system components. The median value of magnitude difference errors, based on measurements by Balega et al. $(2002,2004)$, is about 0.06 .

In frequencies less than the telescope resolution limit, the method appears to reduce the influence of photon bias uncertainties due to, e.g., the weak registration nonlinearity. The atmospheric effect and noisy data can be excluded by selecting a proper spatial frequency range for the fitting algorithm. The method does not need the speckle interferometric transfer function to be corrected by a deconvolution procedure. Thus, no reference star is needed in observations. However, in the case of a close binary $(\rho<2 \lambda / D)$, when the separation and the magnitude difference are correlated, we have to use a reference star. Either a single star or a sufficiently wide binary may be selected as the reference source in this case. In addition, the reference star is useful to look for areas where the transfer function is a constant, which reduces the computer time. The estimation of errors of measurements becomes a trivial procedure.

Examination of our data obtained in various observational sets and the comparison with $\Delta m$ published by other authors demonstrate their high consistency and reliability. 
Table 1. Comparison of $\Delta m_{545}^{\mathrm{c}}$ with the original $\Delta m\left(\mathrm{~B} 2002\right.$, B2004) and HIPPARCOS $\Delta m_{\mathrm{H}}$.

\begin{tabular}{|c|c|c|c|c|c|c|c|c|c|}
\hline HIP & Epoch & $\Delta m_{545}^{\mathrm{c}}$ & $\Delta m$ & $\Delta m_{\mathrm{H}}$ & HIP & Epoch & $\Delta m_{545}^{\mathrm{c}}$ & $\Delta m$ & $\Delta m_{\mathrm{H}}$ \\
\hline & $1990+$ & & & & & $1990+$ & & & \\
\hline 461 & 8.7717 & $1.31 \pm 0.03$ & $1.31 \pm 0.05$ & $1.56 \pm 0.43$ & 26220 & 9.8189 & $3.72 \pm 0.12$ & $3.23 \pm 0.75$ & \\
\hline 689 & 8.7717 & $0.28 \pm 0.05$ & $0.31 \pm 0.05$ & $0.04 \pm 0.39$ & 26926 & 8.7751 & $0.73 \pm 0.05$ & $0.94 \pm 0.12$ & $0.73 \pm 0.31$ \\
\hline 768 & 8.7799 & $1.67 \pm 0.05$ & $1.67 \pm 0.12$ & $1.70 \pm 0.03$ & 27758 & 8.7723 & $1.34 \pm 0.03$ & $1.31 \pm 0.03$ & $0.87 \pm 0.68$ \\
\hline 1392 & 8.7772 & $1.03 \pm 0.02$ & $1.06 \pm 0.02$ & $0.60 \pm 0.27$ & 28832 & 9.8135 & $1.31 \pm 0.03$ & & $1.31 \pm 0.02$ \\
\hline 3795 & 8.7799 & $0.27 \pm 0.14$ & $0.64 \pm 0.04$ & $0.27 \pm 0.05$ & 29269 & 9.8161 & $1.38 \pm 0.02$ & $1.40 \pm 0.03$ & $1.40 \pm 0.03$ \\
\hline 4267 & 8.7798 & $1.06 \pm 0.02$ & $1.11 \pm 0.05$ & $1.05 \pm 0.01$ & 30272 & 9.8216 & $1.11 \pm 0.02$ & $1.29 \pm 0.02$ & $1.090 \pm 0.009$ \\
\hline 4809 & 8.7718 & $0.10 \pm 0.24$ & $0.17 \pm 0.15$ & $0.28 \pm 0.30$ & 34524 & 9.8161 & $1.87 \pm 0.04$ & $1.89 \pm 0.03$ & $1.40 \pm 0.54$ \\
\hline \multirow[t]{5}{*}{4990} & 8.7717 & $0.54 \pm 0.03$ & $0.60 \pm 0.07$ & $0.48 \pm 0.04$ & 40818 & 9.8190 & $0.46 \pm 0.04$ & $0.47 \pm 0.04$ & $0.32 \pm 0.03$ \\
\hline & 8.7744 & $0.51 \pm 0.02$ & $0.56 \pm 0.05$ & & 43861 & 9.8216 & $2.98 \pm 0.15$ & $3.11 \pm 0.19$ & $2.94 \pm 0.08$ \\
\hline & 8.7772 & $0.52 \pm 0.03$ & $0.53 \pm 0.04$ & & 44471 & 9.8217 & $0.38 \pm 0.10$ & $0.28 \pm 0.31$ & $0.39 \pm 0.20$ \\
\hline & 9.8129 & $0.55 \pm 0.02$ & $0.58 \pm 0.06$ & & 46199 & 9.8216 & $2.17 \pm 0.08$ & $2.45 \pm 0.11$ & $2.13 \pm 0.63$ \\
\hline & 9.8155 & $0.57 \pm 0.02$ & $0.53 \pm 0.02$ & & 88136 & 8.7769 & $0.46 \pm 0.05$ & $0.47 \pm 0.04$ & \\
\hline 5531 & 8.7745 & $0.73 \pm 0.03$ & $0.71 \pm 0.03$ & $0.47 \pm 0.18$ & & 8.7769 & $0.42 \pm 0.03$ & & \\
\hline \multirow[t]{2}{*}{6060} & 8.7719 & $0.85 \pm 0.05$ & $0.82 \pm 0.07$ & $0.88 \pm 0.03$ & 90996 & 8.7767 & $0.39 \pm 0.06$ & $0.49 \pm 0.14$ & $1.17 \pm 0.36$ \\
\hline & 9.8209 & $0.90 \pm 0.03$ & $0.89 \pm 0.04$ & & 94076 & 8.7768 & $2.02 \pm 0.04$ & $2.15 \pm 0.05$ & \\
\hline 7580 & 8.7773 & $1.09 \pm 0.02$ & $1.20 \pm 0.03$ & & & 9.8205 & $2.01 \pm 0.03$ & $2.01 \pm 0.05$ & \\
\hline 8258 & 8.7747 & $0.11 \pm 0.13$ & $0.26 \pm 0.08$ & $0.22 \pm 0.07$ & 94679 & 9.8204 & $0.43 \pm 0.03$ & $0.72 \pm 0.02$ & $0.454 \pm 0.007$ \\
\hline 11253 & 8.7747 & $2.76 \pm 0.06$ & $2.79 \pm 0.22$ & $2.58 \pm 0.38$ & 94720 & 8.7767 & $1.47 \pm 0.02$ & $1.47 \pm 0.05$ & \\
\hline \multirow[t]{2}{*}{11352} & 8.7747 & $0.18 \pm 0.06$ & $0.21 \pm 0.06$ & $0.44 \pm 0.34$ & & 9.8205 & $1.42 \pm 0.03$ & $1.42 \pm 0.05$ & \\
\hline & 8.7773 & $0.05 \pm 0.20$ & $0.15 \pm 0.06$ & & 95995 & 8.7769 & $0.26 \pm 0.05$ & $0.33 \pm 0.07$ & $1.39 \pm 0.52$ \\
\hline 11474 & 8.7719 & $0.35 \pm 0.04$ & $0.52 \pm 0.04$ & $0.22 \pm 0.02$ & 96656 & 8.7770 & $0.70 \pm 0.03$ & $0.67 \pm 0.03$ & $0.68 \pm 0.21$ \\
\hline 12640 & 9.8130 & $0.48 \pm 0.02$ & $0.46 \pm 0.02$ & & 97496 & 8.7794 & $0.43 \pm 0.07$ & $0.64 \pm 0.06$ & $0.40 \pm 0.04$ \\
\hline 14075 & 9.8184 & $0.10 \pm 0.13$ & $0.17 \pm 0.16$ & $0.44 \pm 0.22$ & 99257 & 8.7795 & $0.56 \pm 0.04$ & $0.63 \pm 0.04$ & $0.49 \pm 0.03$ \\
\hline \multirow[t]{2}{*}{14230} & 8.7774 & $1.72 \pm 0.04$ & $1.74 \pm 0.05$ & $1.73 \pm 0.21$ & 99874 & 8.7768 & $2.31 \pm 0.11$ & $2.28 \pm 0.32$ & $2.15 \pm 0.07$ \\
\hline & 9.8131 & $1.72 \pm 0.03$ & $1.74 \pm 0.03$ & & 105259 & 8.7770 & $1.40 \pm 0.06$ & $1.52 \pm 0.04$ & 1.13 \\
\hline 14929 & 8.7775 & $0.07 \pm 0.14$ & $0.17 \pm 0.08$ & $0.31 \pm 0.69$ & 105947 & 8.7796 & $1.50 \pm 0.04$ & $1.50 \pm 0.06$ & $1.17 \pm 0.48$ \\
\hline 15309 & 8.7775 & $0.47 \pm 0.03$ & $0.49 \pm 0.03$ & $0.460 \pm 0.005$ & 106393 & 8.7770 & $0.21 \pm 0.08$ & $0.24 \pm 0.07$ & \\
\hline 16025 & 8.7776 & $1.78 \pm 0.03$ & $1.73 \pm 0.05$ & $1.78 \pm 0.14$ & & 8.7795 & $0.19 \pm 0.09$ & $0.20 \pm 0.10$ & \\
\hline 16042 & 9.8185 & $2.82 \pm 0.11$ & $2.77 \pm 0.13$ & $3.15 \pm 0.37$ & 108917 & 9.8127 & $1.48 \pm 0.02$ & $1.47 \pm 0.03$ & \\
\hline 16143 & 8.7749 & $0.55 \pm 0.03$ & $0.63 \pm 0.03$ & $0.36 \pm 0.09$ & 109281 & 8.7742 & $0.51 \pm 0.03$ & $0.50 \pm 0.05$ & $0.39 \pm 0.21$ \\
\hline 16602 & 8.7774 & $0.24 \pm 0.11$ & $0.23 \pm 0.05$ & & 109951 & 9.8152 & $1.92 \pm 0.04$ & $1.87 \pm 0.04$ & $1.83 \pm 0.76$ \\
\hline \multirow[t]{2}{*}{17491} & 8.7746 & $2.69 \pm 0.06$ & $2.69 \pm 0.11$ & & 112695 & 9.8208 & $2.25 \pm 0.05$ & $2.29 \pm 0.08$ & $2.34 \pm 0.36$ \\
\hline & 9.8213 & $2.68 \pm 0.07$ & $2.64 \pm 0.08$ & & 113852 & 8.7744 & $3.36 \pm 0.08$ & $3.38 \pm 0.09$ & $3.04 \pm 0.22$ \\
\hline 17932 & 9.8214 & $1.35 \pm 0.07$ & $1.37 \pm 0.08$ & & 114444 & 8.7771 & $1.83 \pm 0.02$ & $1.83 \pm 0.04$ & $1.85 \pm 0.03$ \\
\hline \multirow[t]{2}{*}{18089} & 9.8187 & $0.28 \pm 0.08$ & & $0.350 \pm 0.009$ & 114576 & 9.8209 & $0.37 \pm 0.03$ & $0.42 \pm 0.07$ & \\
\hline & 9.8213 & $0.28 \pm 0.03$ & & & 115666 & 9.8153 & $0.21 \pm 0.10$ & $0.33 \pm 0.06$ & $0.15 \pm 0.02$ \\
\hline 19009 & 8.7776 & $1.05 \pm 0.08$ & $1.18 \pm 0.15$ & & & 9.8207 & $0.08 \pm 0.18$ & $0.29 \pm 0.06$ & \\
\hline 20087 & 8.7776 & $2.12 \pm 0.12$ & $2.06 \pm 0.17$ & & 116259 & 8.7746 & $2.48 \pm 0.04$ & $2.53 \pm 0.07$ & $2.61 \pm 0.54$ \\
\hline 21280 & 9.8214 & $1.35 \pm 0.03$ & $1.38 \pm 0.05$ & $1.27 \pm 0.71$ & 118287 & 8.7745 & $0.22 \pm 0.09$ & $0.41 \pm 0.04$ & $0.04 \pm 0.02$ \\
\hline 22550 & 8.7722 & $0.15 \pm 0.10$ & $0.35 \pm 0.03$ & $0.24 \pm 0.20$ & & 9.8127 & $0.29 \pm 0.19$ & & \\
\hline 23699 & 8.7750 & $1.36 \pm 0.06$ & $1.44 \pm 0.07$ & $1.48 \pm 0.02$ & & 9.8181 & $0.25 \pm 0.05$ & $0.34 \pm 0.03$ & \\
\hline
\end{tabular}

Acknowledgements. I am grateful to V. A. Vasyuk from the Special Astrophysical Observatory of Russia, who participated in software development at the first stage of the project. I would like also to thank Dr. V. S. Tsvetkova and Dr. V. G. Vakulik from Institute of Astronomy of Kharkov University, and Prof. Y. Y. Balega from the Special Astrophysical Observatory of Russia, for their useful notes. This work has been supported by the Russian Foundation for Basic Research through the grant No. 04-02-17563-a.

\section{References}

Andersen, J. 1991, A\&ARv, 3, 91

Bagnuolo, W. G., Jr., Mason, B. D., Barry, D. J., Hartkopf, W. I., \& McAlister, H. A. 1992, AJ, 103, 1399

Bagnuolo, W. G., Jr., Sowell, J. R. 1988, AJ, 96, 1056

Balega, I. I., Balega, Y. Y., Hofmann, K.-H., et al. 2002, A\&A, 385, 87

Balega, I., Balega, Y. Y., Maksimov, A. F., et al. 2004, A\&A, 422, 627 
Dombrowski, E. G. 1989, BAAS, 21, 1223

HIPPARCOS Catalog, 1997, ESA SP-1200, V. 10

Fabricius, C., \& Makarov, V. V. 2000, A\&A, 356, 141

Goodman, G. W., \& Belsher, J. F. 1976, Proc. SPIE, 75, 141

Hartkopf, W. I., Mason, B. D., McAlister, H. A., et al. 1996, AJ, 111, 936

Hartkopf, W. I., McAlister, H. A., \& Mason, B. D. 2001, AJ, 122, 3480

Henry, T. J., Franz, O. G., Wasserman L. H., et al. 1999, ApJ, 512, 864

Horch, E., Ninkov, Z., \& Franz, O. G. 2001, AJ, 121, 1583

Horch, E. P., Meyer, R. D., \& van Altena W. F. 2004, AJ, 127, 1727

Kuzmenkov, S. G. 1986, SvA, 30, 353

Mason, B. D., McAlister, H. A., Hartkopf, W. I., Griffin, R. F., \& Griffin, R. E. M. 1997, AJ, 114, 1607

Mason, B. D., \& Wycoff G. L. The Second Photometric Magnitude Difference Catalog,

http://ad.usno.navy.mil/ wds/dm2.html
Preibisch, Th., Hofmann, K.-H., Schertl, D., et al. 2000, in Proc. of IAU Symp. 200 on Birth and Evolution of Binary Stars, Potsdam, Germany, ed. B. Reipurth, H. Zinnecker, 106

Prieur, J.-L., Carquillat, J.-M., Ginestet, N., et al. 2003, ApJS, 144, 263

Scholler, M., Balega, I. I., Balega, Y. Y., et al. 1998, Astron. Lett., 24, 283

ten Brummelaar, T. A., Mason, B. D., Bagnuolo W. G., Jr., et al. 1996, AJ, 112, 1180

ten Brummelaar, T., Mason, B. D., McAlister, H. A., et al. 2000, AJ, 119,2403

Turner, N. H., ten Brummelaar, T. A., McAlister, H. A., et al. 2001, AJ, 121, 3254

Turner, N. H., ten Brummelaar, T. A., Roberts, Jr., L. C. 2003, Proc. SPIE, 4839, 1103

Walker, R. L. 1978, BAAS, 10, 410

Woitas, J., Leinert, Ch., Köhler, R. 2001, A\&A, 376, 982

Worley, C. E., Mason, B. D., \& Wycoff G. L. 2001, AJ, 122, 3482 